\title{
Meta-analytical biomarker search of EST expression data reveals three differentially expressed candidates
}

Timothy H Wu', Lichieh J Chu², Jian-Chiao Wang ${ }^{3}$, Ting-Wen Chen ${ }^{2,4}$, Yin-Jing Tien ${ }^{5}$, Wen-Chang Lin ${ }^{1,6}$, Wailap $\vee \mathrm{Ng}^{1,3,7^{*}}$

From Asia Pacific Bioinformatics Network (APBioNet) Eleventh International Conference on Bioinformatics (InCoB2012)

Bangkok, Thailand. 3-5 October 2012

\begin{abstract}
Background: Researches have been conducted for the identification of differentially expressed genes (DEGs) by generating and mining of cDNA expressed sequence tags (ESTs) for more than a decade. Although the availability of public databases make possible the comprehensive mining of DEGs among the ESTs from multiple tissue types, existing studies usually employed statistics suitable only for two categories. Multi-class test has been developed to enable the finding of tissue specific genes, but subsequent search for cancer genes involves separate two-category test only on the ESTs of the tissue of interest. This constricts the amount of data used. On the other hand, simple pooling of cancer and normal genes from multiple tissue types runs the risk of Simpson's paradox. Here we presented a different approach which searched for multi-cancer DEG candidates by analyzing all pertinent ESTs in all categories and narrowing down the cancer biomarker candidates via integrative analysis with microarray data and selection of secretory and membrane protein genes as well as incorporation of network analysis. Finally, the differential expression patterns of three selected cancer biomarker candidates were confirmed by real-time GPCR analysis.
\end{abstract}

Results: Seven hundred and twenty three primary DEG candidates ( $p$-value $<0.05$ and lower bound of confidence interval of odds ratio $\geqq 1.65$ ) were selected from a curated EST database with the application of Cochran-MantelHaenszel statistic $(\mathrm{CMH})$. GeneGO analysis results indicated this set as neoplasm enriched. Cross-examination with microarray data further narrowed the list down to 235 genes, among which 96 had membrane or secretory annotations. After examined the candidates in protein interaction network, public tissue expression databases, and literatures, we selected three genes for further evaluation by real-time GPCR with eight major normal and cancer tissues. The higher-than-normal tissue expression of COL3A1, DLG3, and RNF43 in some of the cancer tissues is in agreement with our in silico predictions.

Conclusions: Searching digitized transcriptome using $\mathrm{CMH}$ enabled us to identify multi-cancer differentially expressed gene candidates. Our methodology demonstrated simultaneously analysis for cancer biomarkers of multiple tissue types with the EST data. With the revived interest in digitizing the transcriptomes by NGS, cancer biomarkers could be more precisely detected from the ESTs. The three candidates identified in this study, COL3A1, DLG3, and RNF43, are valuable targets for further evaluation with a larger sample size of normal and cancer tissue or serum samples.

\footnotetext{
* Correspondence: wrng@ym.edu.tw

'Institute of Biomedical Informatics, National Yang Ming University, Taipei,

Taiwan, R.O.C

Full list of author information is available at the end of the article
}

\section{Ciomed Central}

(c) 2012 Wu et al.; licensee BioMed Central Ltd. This is an open access article distributed under the terms of the Creative Commons Attribution License (http://creativecommons.org/licenses/by/2.0), which permits unrestricted use, distribution, and reproduction in any medium, provided the original work is properly cited. 


\section{Background}

One of the key aspects in the study of cancer is to understand the principles and mechanisms of gene expression variation contributing to cancer genesis and progression. The identification of genes differentially expressed between normal and cancer cells/tissues is not only helpful for designing diagnostic and therapeutic procedures, but also for understanding cancer biology as a whole. In this regard, DNA microarrays have been the dominating platform in the high-throughput study of cancer transcriptomes since their emergence in the mid-1990s [1,2]. However, there are several drawbacks, which include: high background level signals resulting from cross-hybridization $[3,4]$; difference in hybridization properties due to different probe sequences; limited dynamic range due to background level and saturation, and difficulty in detecting splicing isoforms and unknown genes. For these reasons, with the advancement of the next generation sequencers, we are seeing high-throughput transcriptome mapping and quantifying method, also known as RNA-Seq, to begin to supersede microarray in expression profiling. However, RNA-Seq experiments are relatively demanding in terms of time, cost, and computation equipment. Experimental differences between different sequencing platforms may complicate transcriptome analysis with multiple tissue sources. Since exploring meta-analysis from traditional digital expression data such as EST derived from cDNAs [5-8] is more feasible, this study may serve as a precursor to more complicated experiments.

Originally primarily aimed for cataloging of transcript repertoire, ESTs from large-scale cDNA sequencing projects such as Cancer Genome Anatomy Project (CGAP), Human Cancer Genome Project (HCGP), and Cancer Genome Project (CGP) also allow searching for differentially expressed genes (DEGs) in specific tissue types or in whole genomes [9-11]. Several in silico analysis tools such as NCBI Unigene cDNA xProfiler [12], CGAP Digital Differential Display (DDD) [13], and CGAP Digital Gene Expression Displayer (DGED) [14] are available online allowing the analysis of publicly available data. While standard statistical methods such as Fisher's exact test for finding DEGs in two-class problems (e.g. cancer vs. normal) or Pearson's correlation are commonly used [9], there are also specially developed methods for finding DEGs in the landscape of digital signals for two-library problems $[15,16]$ or for multiple libraries [17]. The online tools as well as the statistical methods remain useful to this day in EST or even RNA-Seq projects [18-23]. Aside from searching for DEGs, the searches for gene transcript isoforms specific to particular libraries were also demonstrated and many of these attribute differentially expressed isoforms to human cancers [24-31].

In spite of the successful applications, these tools or methods are not without limitations. xProfiler reports differential expression in an all-or-none manner where only a list, but not statistical quantification, of candidates is reported. DDD allows quantification using Fisher's exact test. However, the nature of the test dictates that comparisons of three or more libraries involve multiple pair-wise comparisons, and thus there are no easy comparisons of library specific genes. DGED uses a Bayesian approach to find DEGs, but it is also pair-wise. The reported "odds ratio" is perhaps better described as "relative risk" and may be biased with unequal sampling. Another popular and useful Bayesian-based method originally developed for EST analysis by Audic and Cleverie [15] is also popular for RNA-Seq data. It is less conservative than Fisher's exact test, but it also does not apply to multi-class problems. The multi-class comparison method established by Stekel et al. [17] finds specificity in one condition out of all and is useful in application such as finding DEGs in multitissue libraries. However, in the search for cancer DEGs, a subsequent analysis of differential expression between cancer and normal libraries of the tissue of interest may not yield fruitful results due to the possible scarcity of EST sampling in the particular tissue type. On the other hand, the naïve method of pooling all data into the two-class problem of normal versus cancer when searching for differentially expressed genes or differentially splice variants [27] risks introducing bias. In extreme cases, one may encounter the fallacy of Simpson's paradox [32] where genes in reality more active in the normal condition appear to be more so in the cancer condition (discussed later in this paper).

We now report on the application of a computational and integrative approach to analyze cancer differentially expressed genes (DEGs). The statistical method we employed is Cochran-Mantel-Haenszel statistics (CMH) [33] and to the best of our knowledge has not been applied in this context. Instead of pooling all normal and all cancer ESTs from different tissue types to fit into a two-class problem as by using the 2 by 2 contingency $\chi^{2}$ test or the Fisher's exact test, $\mathrm{CMH}$ allows original stratification of libraries in their respective tissue types, yet exhaustively analyzes expression between cancer and normal conditions across all tissue types. The method is an extension to $\chi^{2}$ test which, in our application, measures the association between cancer and gene expressions, adjusting for the tissue confounding factor. This approach allows one to find genes that are overall differentially expressed in cancer, or multiple-cancer genes, irrespective to a specific tissue type. The method is demonstrated in this paper to exhaustively analyze ESTs from the dbEST database [34]. To the best knowledge of the authors, such an all-inclusive, wholetranscriptome analysis has not been redone in recent years now that more ESTs than ever are available.

Our filtering of EST libraries was also more rigorous than many previous studies. Notably, we excluded the 
ORESTES (open reading-frame EST sequencing) libraries [35] on which a normalization procedure had been applied. Libraries from cell line were also excluded owning to their unrepresentativeness of primary cancer cell transcriptomes. Our analysis pipeline further focused on enrichment of the DEGs by cross examination with expression data of a different platform, i.e. the microarray data, and selecting for membrane and secretory associated protein genes since we intend to find therapeutic targets or biomarkers, and conducting STRING (The Search Tool for the Retrieval of Interacting Genes) network analysis to show the cancer enriched clusters [36]. With real-time qPCR validation, we have identified three candidates that are inclined to express in cancer across more than one tissue types. We hope such a meta-analytical and multiple-tissue comparison can serve as an exploratory experiment for future multi-library or multitissue study of other digital sources such as RNA-Seq.

\section{Methods}

\section{Overview}

Our approach was to exploit the entire collection of human EST sequences from dbEST [34] to obtain transcripts from different type of cells/tissues/organs. The assumption was that the activities of the genes can be represented by their transcripts, and also reflected by the number of representing ESTs in the NCBI dbEST database, given that a large number of mRNAs (cDNAs) were sequenced. Pertinent sequences from different sources were matched to genes and tallied together. Through the annotation of each EST record, we obtained the tissue type and condition type (normal or cancer) from which it was derived. With the information, we then had the entire gene transcription profile for all the tissues and conditions. Next, cross examining data of other sources including microarray data, secretory and membrane associations as well as analyzing protein associations with STRING [36] allowed us to narrow down the list of candidate genes. The process is illustrated in Figure 1.

\section{Human gene reference sequence preparation}

The NCBI Reference Sequences (RefSeq Release 38, November 11, 2009) [37] were downloaded from its ftp site [38]. Homo sapiens RefSeq records were selected and subjected to repeat masking via RepeatMasker [39].

\section{Human EST sequence preparation and library filtering}

Human EST data (Released on December 11, 2009) and their cDNA library information were downloaded from NCBI dbEST database [34] and CGAP [40]. Program in Python language was written to mark for discard the unsuitable libraries when the keywords such as "enrichment", "subtract", "pcr", and "normalized" were found in the DESCR, UNIQUE_PROTOCOL, or KEYWORDS fields of the library information. An arbitrary cutoff of $>400$ was chosen to the highly unrepresentative libraries (approximately 7,000 libraries constituting approximately 650,000 ESTs were discarded as a consequence). To curb from incorrect inclusions or exclusions, we finalized the process with manual curation. Libraries made from mixed tissues or cell lines were also discarded. The final libraries from CGAP were manually classified into 48 different tissue types and two different conditions, normal and cancer.

\section{EST to gene assignment}

The BLAT alignment tool was used to align ESTs to RefSeqs as a mean to assign ESTs to genes [41]. The criteria of having an identity of $95 \%$ or above and the minimum length of 100 nucleotides were set for a match. The RefSeq match with the highest identity was assigned for the EST. If two RefSeq matches shared exactly the same identity, the program chose the first encountered.

\section{EST count and summarization}

The procedure attributes each transcript represented by RefSeq its expression profile across different tissue and condition types based on EST assignment counts. Each EST has its corresponding tissue type and condition type classification, based on its source clone library. For example, a transcript with an aligned EST from a lung cancer clone library is one expression count each in tissue type lung and condition type cancer. This way, after all ESTs were counted, each transcript has a profile of expression across various libraries and conditions. Expressions from different transcript variants of the same gene were pooled to obtain a single gene expression. The raw counts were thus made into transcription profile for each gene for further statistical analysis.

\section{Statistical evaluation of cancer candidates}

Cochran-Mantel-Haenszel statistics (CMH) was applied to evaluate cancer differential expression of each gene. To evaluate each gene, other genes were pooled as "other genes" to create a $2 \times 2 \times k$ table consisting of data from tissue-condition cross, where $k$ was the number of tissues $\times 2$ (two conditions). A contrived example of $2 \times 2 \times k$ table where $k$ is 2 is shown in Table 1 . Gene $A$ is the gene under study while other genes are pooled together as "other genes". Only Tissue I and Tissue II columns are calculated in $\mathrm{CMH}$. The pooled ones are not part of the analysis. Akin to Fisher's exact test, the test assumes that "other genes" should consist mostly of genes not differentially expressed between normal or cancer conditions. Or, some of them are DEGs for one condition, but they are at least partly canceled out by DEGs for the other. In any case, the imbalances of cancer counts to normal counts in the second row is regarded as owning to sample bias and it serves 


\section{EST lib selection}

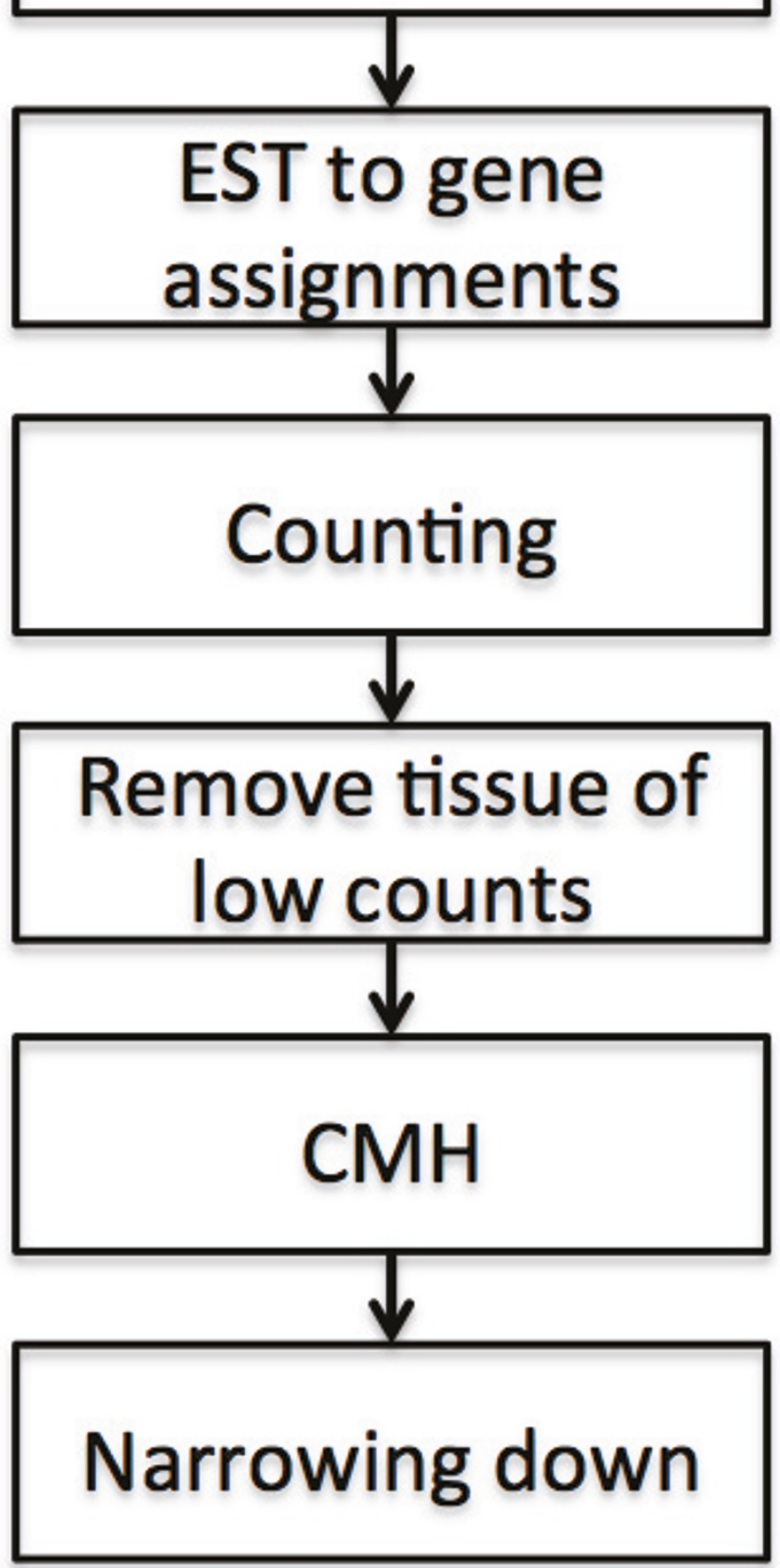

Figure 1 The basic steps in searching for differential expression genes. EST library selection involves selection of suitable EST clone libraries, EST to gene assignment, counting the results, remove tissue categories with low counts, statistical analysis with CMH and the narrow-down of differentially expressed genes (DEGs). The narrow-down procedures includes cross referencing with public microarray data, annotating membrane and secretory proteins, analyzing with String network, and for a few selected genes, validate the expression in different tissues by RTqPCR. 
Table 1 A hypothetical EST count table demonstrating CMH analysis and also a contrived example of Simpson's paradox.

\begin{tabular}{ccccccc}
\hline & \multicolumn{2}{c}{ Tissue I } & \multicolumn{2}{c}{ Tissue II } & \multicolumn{2}{c}{ Pooled } \\
\cline { 2 - 7 } & Normal & Cancer & Normal & Cancer & Normal & Cancer \\
\hline Gene A & 280 & 580 & 20 & 20 & $\mathbf{3 0 0}$ & $\mathbf{6 0 0}$ \\
Other genes & $\mathbf{2 0 , 0 0 0}$ & $\mathbf{8 0 , 0 0 0}$ & $\mathbf{3 8 0 , 0 0 0}$ & $\mathbf{6 2 0 , 0 0 0}$ & 400,000 & 700,000 \\
\hline
\end{tabular}

This hypothetical case serves both as an example of how Cochran-MantelHaenszel (CMH) is applied as well as the occurrence of Simpson's paradox. Gene A is the gene under investigation. Expressions from all other genes are pooled into the "other genes" row. Bold typeface indicates columns showing higher cancer vs. normal propensities. CMH is applied on the stratified tissue columns (but not on the pooled data). A casual observation involving only the pooled data would suggest Gene $A$ as having higher expression in cancer $\left(X^{2}\right.$ test $p$-value close to 0 when analyzing only the pooled). However, a closer inspection on each of the tissue columns reveals otherwise. The observed difference between cancer and normal of the "other genes" is theoretically mostly due to sampling bias.

as a metric against which Gene A is measured. By continuously isolate values for gene currently under study while pooling all other genes to the second row, an odds ratio and a confidence interval is calculated for each gene. Genes with a p-value $<0.05$ and an lower bound of confidence interval of odds ratio $\geqq 1.65$ are selected for further analyses.

\section{Microarray cross reference}

Human U133 Plus 2.0 GeneChip array CEL data were downloaded from Gene Expression Omnibus (GEO) [42]. When computing power allows, the data were processed with AffyPLM [43] using its three-step procedure of processing background signals with GCRMA, normalizing signals with quantile normalization, and summarize probe signals with medium polish. For large experimental datasets that were computationally infeasible for us, we used justRMA from the Affy package [44]. For experimental dataset without raw CEL data, we obtained the preprocessed matrix files via GEOQuery [45]. Regardless of the source of array signal processing, we analyzed the genes for differential expression with Limma [46]. Differentially expressed gene candidates with p-value $<0.05$ and $\operatorname{logFC}>1.0$ were selected and crossed with genes from EST profiling with statistical evaluation. For each array, the significant genes were crossed with our EST profiling results. The union of these intersecting genes was selected for further evaluation.

\section{Annotation of secretory proteins}

To identify our differentially expressed genes with secretory annotation, a list of 3,975 proteins with secretory annotation originated from the conglomeration of data from Uniprot (1,632 unique proteins) [47], Human Plasma Proteome Organization (HUPO) (889 proteins), and Secreted Protein Database (SPD) (4,142 proteins) [48]. This list was matched against DEGs to give them secretory annotation.

\section{Annotation of membrane proteins}

Membrane protein annotations were gathered from five sources - TOPDB (283 proteins) [49], LOCATE (2629 proteins) [50], PDB_TM (41 proteins) [51,52], OPM (107 proteins) [53], and MPDB (23 proteins) [54] - to generate a unique list of 2,767 membrane proteins. Any DEGs on this list would confer it a membrane annotation.

\section{Validation of tissue expression profiles of candidate genes}

TissueScan ${ }^{\mathrm{TM}}$ Cancer Survey Panel 96-I qPCR array panel (Origene Technologies, Rockville, MD) containing the cDNAs of 3 normal and 9 cancer tissues each from 8 organs (breast, colon, kidney, liver, lung, ovarian, prostate, and thyroid) was used to examine the expression profiles of selected cancer differentially expressed gene candidates. Real-time qPCR analyses with the Taqman ${ }^{\circledR}$ Gene Expression Assay kits (Applied Biosystems, Foster City, CA) and FAM- and VIC-labeled target genes and HPRT1 internal control primers, respectively, were performed according to the manufacturer's suggested procedure on an Applied Biosystems Prism 7500 system. Relative specific gene expression was quantified by normalization against the HPRT1 with the $\triangle C T$ method. Gene expression changes were quantified as $2^{-}$(CT gene - CT control).

\section{Results}

Human ESTs selection and tissue distribution

The basic steps of our analysis are illustrated in Figure 1. A total of 8,296,089 human EST sequences (Dec. 11, 2009 release) were downloaded from the NCBI. Despite the size of the data, not all ESTs are relevant for our gene expression analysis. After screening the 8,907 EST libraries as described in the methods section above, 8,447 unsuitable libraries, the preparation of which involved PCR amplification, normalization, subtraction, etc. or originated from cell lines, were discarded. The remaining 460 libraries consisted of 2,386,536 EST sequences representing approximately a third of all the downloaded human ESTs.

After BLAT alignment of the 2,386,536 ESTs to 44,513 gene transcripts from RefSeqs, approximately 1,644,960 (68.92\%) ESTs with at least 100 nucleotides matched to RefSeqs were detected. An examination of the sources of the matched ESTs indicated that the representativeness of each tissue is skewed and that the brain is the most represented out of all tissues. Among the 48 different tissues, brain ESTs constituted 26\% of all matched ESTs, uterus $(6.40 \%)$ ranked second, followed by testis (5.91\%), placenta (4.33\%), pancreas (3.99\%), muscle (3.88\%), liver (3.51\%), kidney (3.52) and others each below 3\% (see Additional file 1). Similarly, condition type (normal and cancer) representation was also skewed. Normal tissue type had 1,251,883 ESTs combined, and cancer tissue had 393,077 ESTs in the ratio of roughly 3 to 1 . Originally before 
filtering out those from the cell lines, there were more cancer ESTs and the ratio of normal ESTs to cancer ESTs was roughly 1 to 3 . This showed how much more rigorous our filtering was. Unfortunately, this also meant we had a much smaller dataset to work with.

The unequal distribution of the 1,644,960 matched ESTs in different tissue types caused some tissue types to be ill-represented. For example, the number of brain EST hits dominated over other tissue types. On the other hand, spinal cord had the least count with 430 EST hits. The latter had little value for our application. Therefore, we only took a tissue type into consideration when its total EST hit count was above the cut-off of 20,000. Considering that the human genome has approximately 22,000 genes, the cut-off still did not allow "deep" probe into gene expression. Nevertheless, the method we employed did not attempt to identify specific gene expression in one particular tissue; therefore, the problem was mitigated.

We also categorized ESTs according to their clone library classification, to either be from normal or from cancer. Sometimes a certain tissue-condition type was so under-represented that the information was not trustworthy. For example, adipose had 10,362 normal hits but only 440 cancer hits, and heart tissue had 22,179 normal hits but no cancer hits. For these cases, data was kept throughout the analysis. But these data did not make contribution to our analysis.

Since our EST assignments were made to transcripts represented by RefSeq sequences, when the entire assignment procedure was done, each transcript variant had its expression profile across all tissue-condition types. Due to the lack of enough ESTs data, differentiating between different splicing variants of the same gene was not feasible. We had to pool expression from different splicing variants into a single expression profile representing the gene.

\section{Analysis of differentially expressed genes}

Due to the small sample size (EST counts), it was only realistic to evaluate gene expression based on all ESTs of all tissues. However, tissue type was a confounder. If all counts for each gene were pooled as "normal" or "cancer" regardless of the tissue of origin, the count would be incorrect. To solve both the sample size and the tissue confounder problems, Cochran-MantelHaenszel statistical method was employed to identify genes with differential expression as described in the method. We used the arbitrary cut-offs of p-value < 0.05 and odds ratio $\geqq 1.65$ to obtain a primary set of candidates. As a result, a total of 723 cancer differentially expressed gene candidates were selected. The 1.65 cut-off is chosen based on a good coverage to a list of well-known biomarkers or genes known to associate with cancer (Table 2).

To show that this list of 723 genes was enriched for cancer and thus obtains credibility for our methodology, we looked for cancer related pathways associated with them in GeneGO [55] pathways, which covered 650 signaling and metabolic networks (Figure 2A). Among the 10 most significantly matched pathways, several are cancer related - Pathways number 1, 3, and 4 involve immune response; number 2 and 5 involve cytoskeleton remodeling; number 6 is transition and termination of DNA replication; and number 8 and number 9 are adhesion related. In addition, the result of GeneGo disease enrichment analysis (Figure 2B) indicates our set of genes as neoplasm enriched: seven out of the 10 most associated diseases are related to cancer. The disease ranks the highest is neoplasms, followed by neoplasm by site, and digestive systems neoplasm. This list reveals that our 723 DEGs covers general neoplasm related functions, and not specific to any particular neoplasm, as digestive, urogenital and breast are all covered.

Table 2 EST counts and odd ratios of 11 well-known cancer-related genes present in our list of DEGs.

\begin{tabular}{|c|c|c|c|c|c|}
\hline $\begin{array}{l}\text { Gene } \\
\text { symbol }\end{array}$ & Description & Total & Normal & Cancer & $\begin{array}{l}\text { Odds } \\
\text { ratio }\end{array}$ \\
\hline BCAN & Homo sapiens brevican & 391 & 79 & 312 & 10.4 \\
\hline KRT14 & Homo sapiens keratin 14 & 205 & 40 & 165 & 9.1 \\
\hline KRT16 & Homo sapiens keratin 16 & 41 & 7 & 34 & 7.8 \\
\hline MMP11 & Homo sapiens matrix metallopeptidase 11 (stromelysin 3) & 68 & 20 & 48 & 5.3 \\
\hline MUC1 & Homo sapiens mucin 1, cell surface associated & 69 & 30 & 39 & 4.2 \\
\hline VEGFA & Homo sapiens vascular endothelial growth factor A & 82 & 33 & 49 & 3.7 \\
\hline AGRN & Homo sapiens agrin & 503 & 143 & 360 & 3.5 \\
\hline COL3A1 & Homo sapiens collagen, type III, alpha 1 & 145 & 90 & 55 & 3.5 \\
\hline MMP1 & Homo sapiens matrix metallopeptidase 1 (interstitial collagenase) & 70 & 29 & 41 & 3.3 \\
\hline EGFR & $\begin{array}{l}\text { Homo sapiens epidermal growth factor receptor (erythroblasticleukemia viral (v-erb-b) oncogene } \\
\text { homolog, avian) }\end{array}$ & 49 & 79 & 312 & 10.4 \\
\hline AFP & Homo sapiens alpha-fetoprotein & 391 & 40 & 165 & 9.1 \\
\hline
\end{tabular}




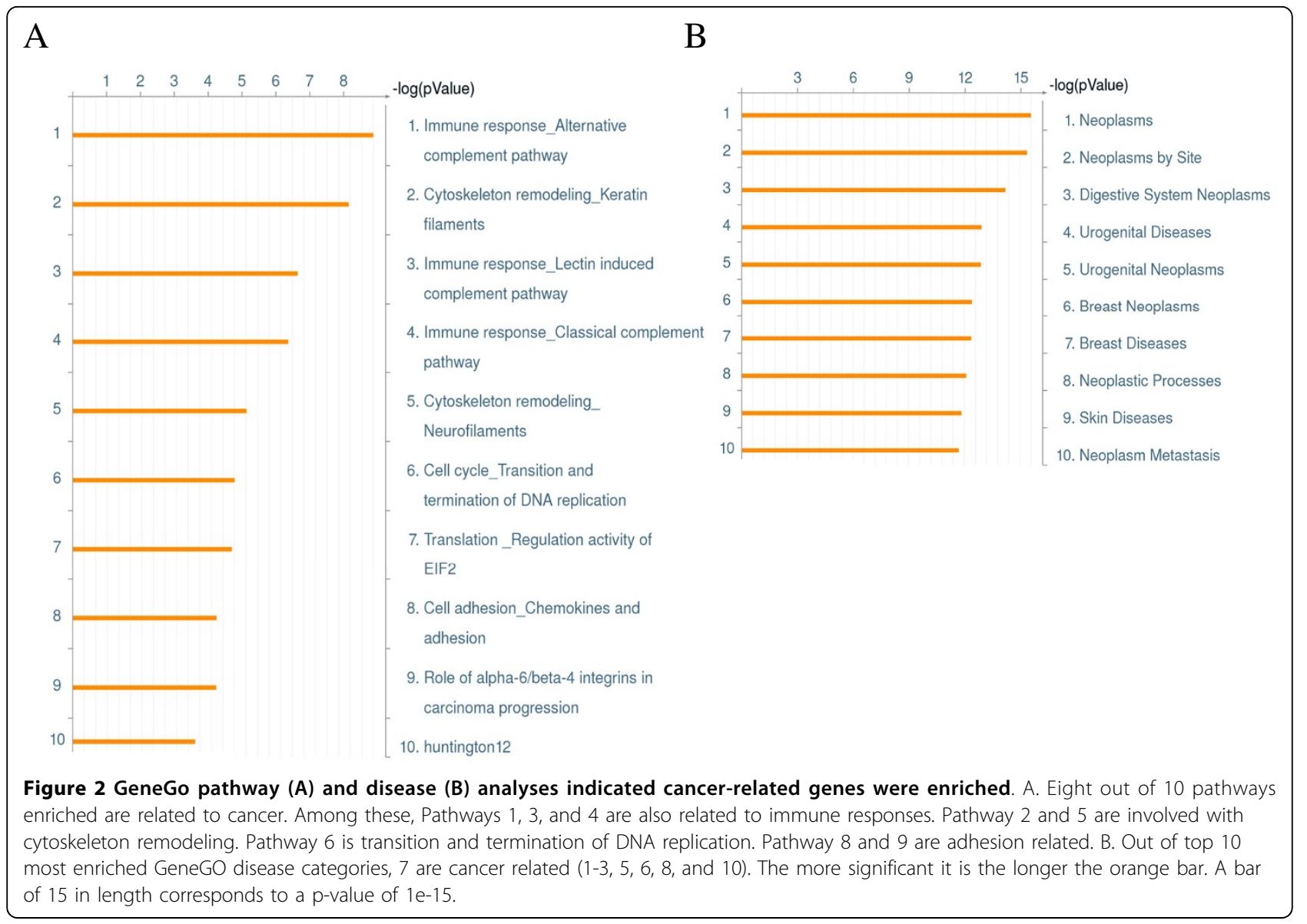

To narrow down this list of biomarkers, we crossed examined the expression profiles of the candidates with the differentially expressed genes in 6 microarray experiments, i.e. two each of ovary and uterus, and one each of pancreas and colon (Table 3). These tissue types were selected based on the following reasons. We noticed that many of our candidate genes had the most expression in ovary tissue (after normalization). The other concern was the number of ESTs. Since our candidate genes were derived from EST sampling of various tissue types, they

Table 3 Five microarray projects cross referenced with our set of 723 DEGs

\begin{tabular}{lcccc}
\hline GEO & $\begin{array}{c}\text { Tissue } \\
\text { type }\end{array}$ & $\begin{array}{c}\text { Test sample size (n } \\
\text { vs. c) }\end{array}$ & $\begin{array}{c}\text { Sig genes } \\
\text { DN }\end{array}$ & Reference \\
\hline GSE18520 & Ovary & 10 vs. 53 & 79 & {$[66]$} \\
GSE14407 & Ovary & 12 vs. 12 & 109 & {$[67]$} \\
GSE764 & Uterus & 4 vs. 7 benign & 0 & Unpublished \\
GSE764 & Uterus & 4 vs. 8 malignant & 2 & Unpublished \\
GSE15471 & Pancreas & 39 vs. 39 & 120 & {$[68]$} \\
GSE23878 & Colon & 24 vs. 35 & 74 & Unpublished \\
\hline
\end{tabular}

n: normal, c: cancer

GSE764 has two entries since we compared pair-wise between normal vs. benign and normal vs. malignant. were influenced more heavily by tissue types with more EST representation due to deeper sampling from them. Therefore, the rest of the tissue types were selected based on their representativeness. Of the 723 DEGs, 235 candidates were also found to be differentially expressed genes in our microarray analysis.

Since membrane and secretory proteins could be potential therapeutic target or serum biomarkers, the subcellular location of the 235 DEGs were examined against the secretory and membrane protein lists consolidated from public databases. Among these, 96 DEGs were putative membrane or secretory proteins - 57 had only secretory annotation, 27 had only membrane annotation and 12 had both.

\section{Literature search and STRING analysis of the 96 DEGs}

To further examine whether the 96 membrane/secretory DEGs identified in our EST database mining had enriched cancer-related genes, we searched the literatures for known associations with cancers. In additions, they were also analyzed with STRING for interactions, which are based on experimental evidence or prediction, such as conserved genomic neighborhood, gene fusion, co-occurrence across genomes, pathways, protein complex, co-regulation, or 
other literature sources such as co-mentioning. The network of the STRING interactions of the 96 DEGs together with the literature search results were plotted based on the combined STRING score with Cytoscape [56] (Figure 3). Approximately 68 proteins formed a big cluster of interacting proteins and a large proportion of the DEGs $(88 \%)$ had published cancer association with clinical or non-clinical experimental supports. This demonstrates the value of our integration strategy since we had an ample of literature supports.

The 96 DEGs were selected out of their general cancer propensity without necessarily referring to any particular tissue type. However, we can still assess the general tissue distributions shown in Figure 4. A gene has a tissue representation if any EST from a clone library of the tissue type is matched to it. We can see that some genes are observed across many tissue types. A gene could be observed across a variety of tissue types if it is pan-tissue, and its expression measure is relatively abundant. Separately, Woolf's test for heterogeneity can also give hints to whether a gene is pan-cancer. Those that were found as significant in this test were considered having unequal representation in different genes; although whether they are pan-cancer require further evaluation.

\section{Three candidates had higher expression in several cancer tissues}

Three cancer differentially expressed secreted protein gene candidates, COL3A1 (Collagen alpha-1(III) chain), DLG3 (Discs large homolog 3), and RNF43 (Ring finger protein 43 ), which had an odds ratio of $3.55,7.97$, and 4.03, respectively, and with limited or no clinical support were selected for real-time qPCR analysis using the Taqman ${ }^{\mathbb{B}}$ Gene Expression Assay kits (Applied Biosystems, Foster

\section{String (network analysis) and literature search of the 96 genes}
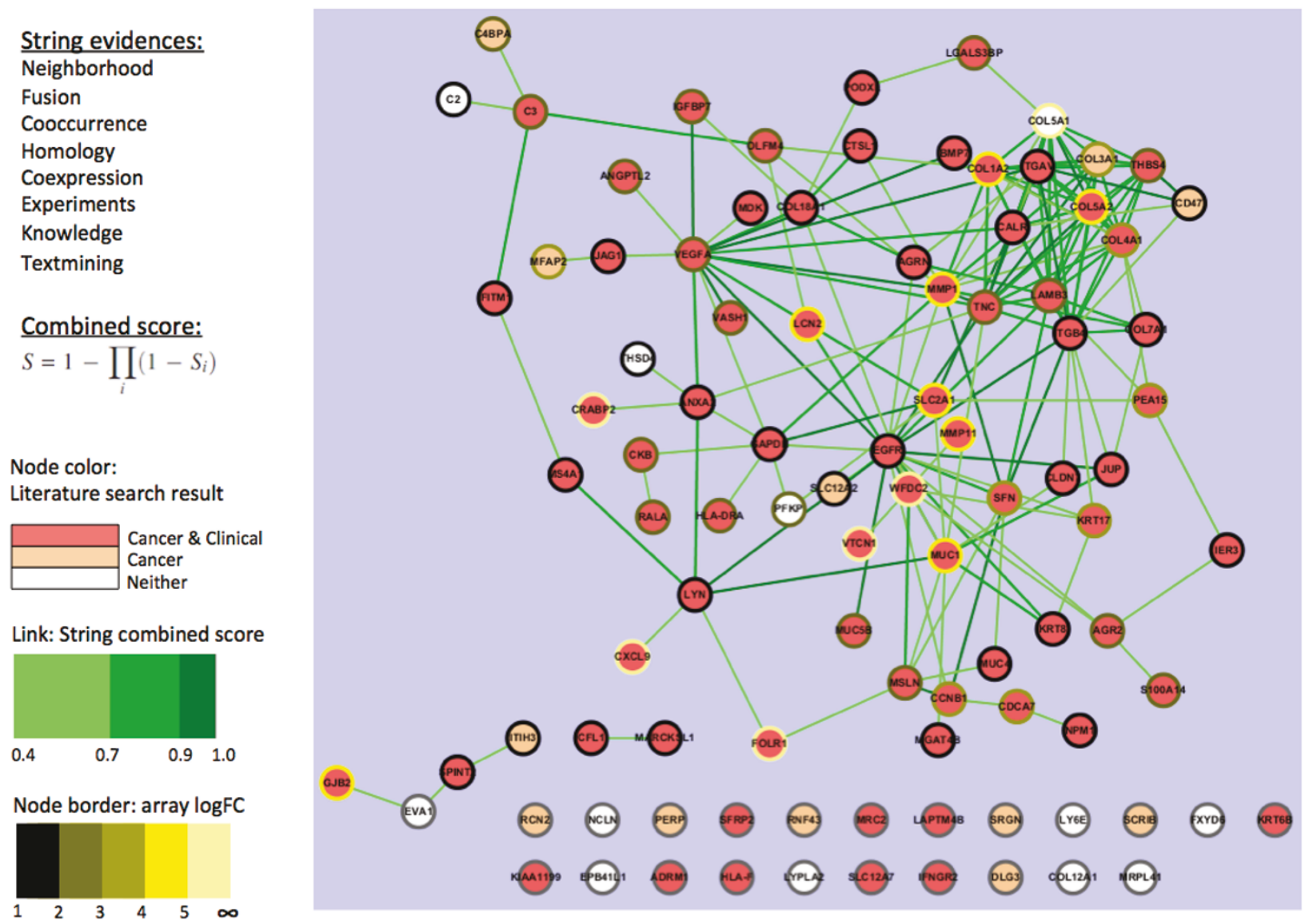

Figure 3 STRING analysis and literature reviews indicated most candidate genes are highly connected and have published cancer associations. STRING analysis is based on several evidences indicating the strength of relationships between pairs of proteins. The STRING output was imported into Cytoscape to depict the network. Darker link denotes stronger relationships. Node colors red, apricot, and white denotes literature search with clinical cancer reports, cancer report but not from clinical samples, and no literature search support found, respectively. Lighter border denotes greater logFC of cancer over normal expression in microarray. 


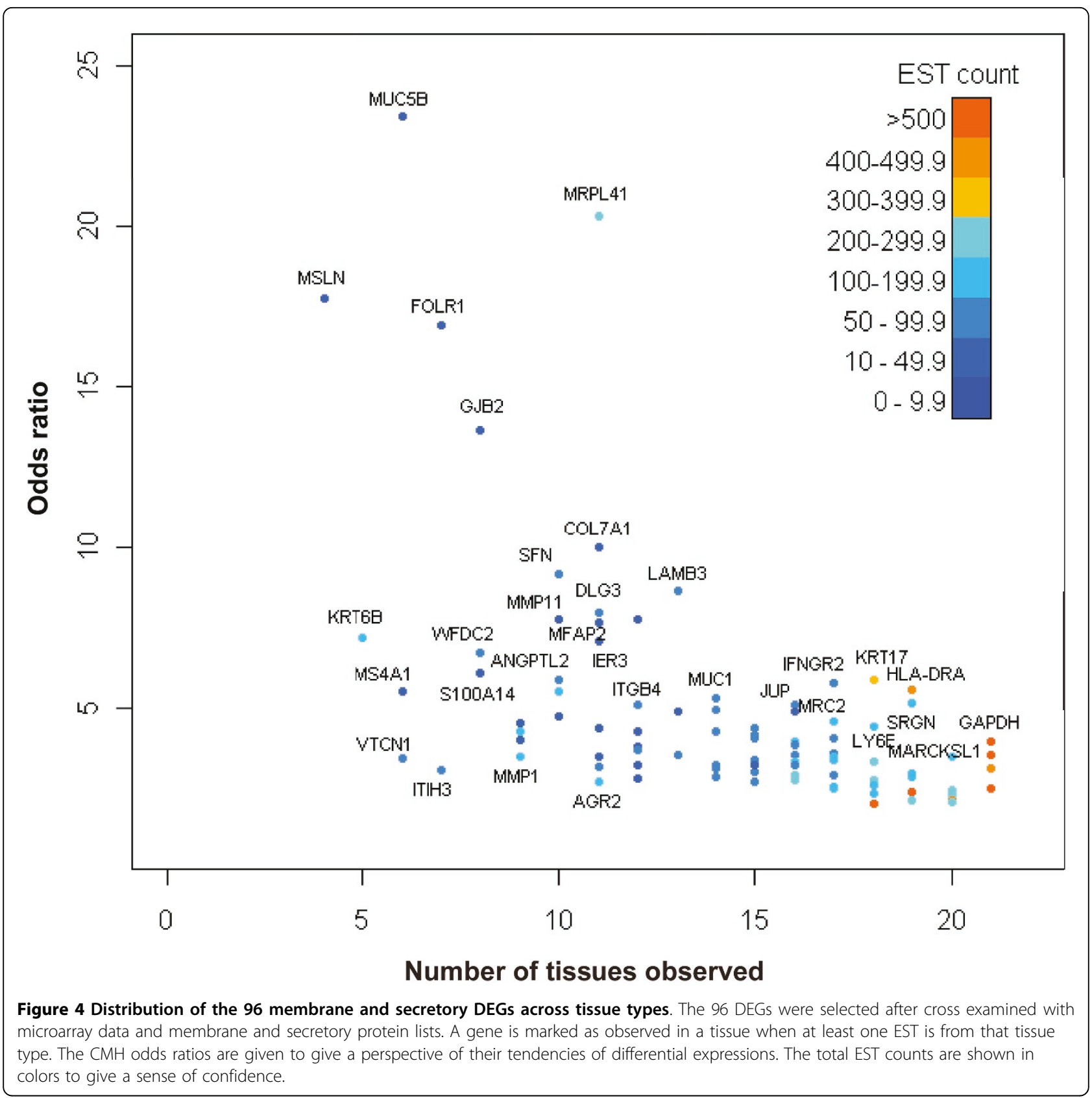

City, CA) (Figure 5). With the HPRT1 as the reference, higher expressions of these genes were noticed in at least some of the cancer tissues. Apparently, the average relative expression levels of COL3A1 in breast, liver, thyroid cancer samples were higher than their normal counterparts. The average expression levels of DLG3 in breast, kidney, liver, lung, and ovarian cancers, and RNF43 in colon, liver, lung, ovarian, and prostate cancers were also found to be higher than their normal tissues. The expression of COL3A1 in approximately 5 of the liver cancers, DLG3 in 5 of the liver, 7 lung and 5 ovarian cancers, and RN43 in 7 of the colon, 8 ovarian and 5 prostate cancers seemed to have higher expressions than the normal tissues. In light of the limited sample size, the three candidates appear to have an overall higher expression in cancer tissues.

\section{Discussion}

Reported here is an integrative, meta-analytic approach for the discovery of pan-cancer differentially expressed gene candidates. Our primary enrichment included a set of 723 DEGs with cancer associations supported by GeneGO disease and pathway analysis. Further integrative evaluations with cancer differentially expressed genes suggested by microarray data narrowed the list down to 234 genes, and 


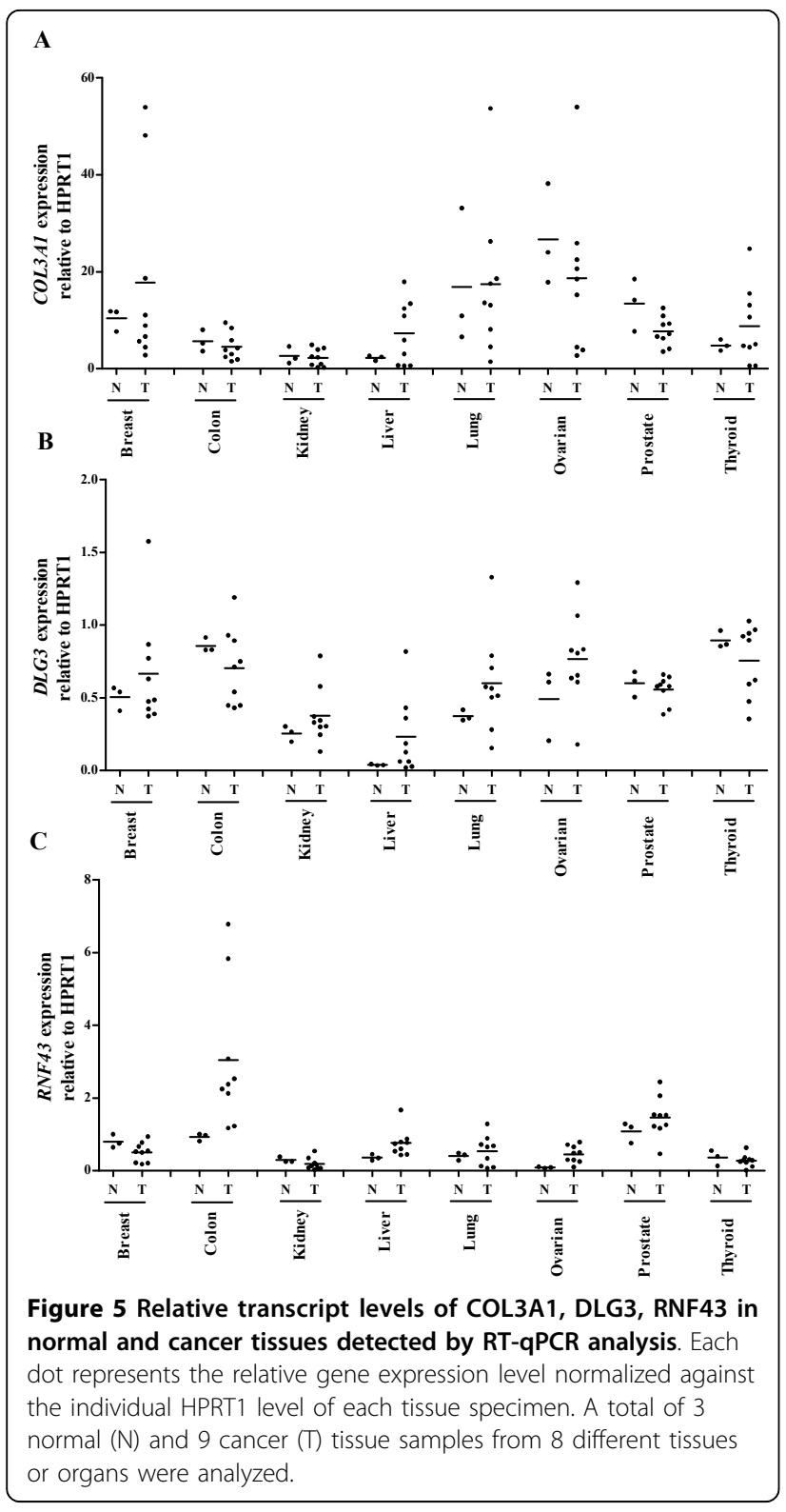

among these there were 96 DEGs likely belonged to either secretory and membrane protein genes. Further STRING protein network analysis and literature reviewing indicated $71 \%$ of the 96 DEGs were highly connected and many of them were associated with cancers in previous publications.

\section{Simpson's paradox}

The meta-analytic nature of our study brought us the opportunities as well as challenges to study the digital signatures of various transcriptomes in a new perspective. Comparing to experimental methods that focus on a single tissue type or limited tissue types, our approach allows us to find genes inclined to express in cancer in a pan-tissue manner. An important challenge of our approach is to avoid Simpson's paradox which can occur in a meta-analysis study [32]. Simpson's paradox is where the association between two variables may show a correlation that is reversed in direction from what is observed from stratified subgroups. A contrived example is shown in Table 1 in which gene A appears to have a higher cancer expression when pooled, but it is in fact not so under individual stratified sub-tables. This may be somewhat of an extreme case, where directionality of the ratios actually differs between the subtables and the pooled table. However, the tissue confounder still introduces bias, large or small, that may throw our judgment off. In this study, we used CMH to analyze the data based on stratified sub-tables to avoid running into this paradox. One could also analyze only one tissue at a time for differential expression, but this means one has a smaller dataset to work with. $\mathrm{CMH}$ could avoid this problem since it uses EST counts from all tissues instead of analyzing just the normal and cancer propensity under each individual tissue type.

In our actual data, the odds ratio of the pooled table is also different from that of the stratified table. For example, the gene PTRF, a polymerase I and transcript release factor, has a pooled odds ratio of 0.40 and a CMH odds ratio of 0.16 calculated from stratified sub-tables. In this particular case, both odds ratios indicated an inclination toward a higher normal expression and are both statistically significant although at different degree (the pooled has a p-value of $5.269 \mathrm{e}-15$ under a $2 \times 2 \chi^{2}$ test [57] versus CMH's 7.16E-77). For the gene VCAN (versican), the pooled odds ratio is 1.86 and $\chi^{2}$ test yields a significant $\mathrm{p}-$ value of $1.83 \mathrm{e}-4$. However, $\mathrm{CMH}$ gives an insignificant result for this gene with p-value of 0.25 . As an extreme case, GBP6 (guanylate binding protein family, member 6) has a pooled odds ratio of 6.69 and $\chi^{2}$ test gives a p-value smaller than 2.2e-16 (approaching 0), whereas with $\mathrm{CMH}$ the odds ratio is 0.73 , actually indicating a higher normal counts, although CMH p-value of 0.15 is insignificant. This indicates Simpson's paradox in action. Careful inspection showed that all cancer counts and most normal counts of GBP6 were contributed by the tongue tissue source. Out of a total of 50 cancer counts and 21 EST normal counts, tongue accounts for cancer and normal counts of 50 and 17, respectively. For this gene, the tongue cancer count 50 is not influential under a total of 29,479 cancer counts and 7,486 counts for the tongue. Thus pooling loses information in this respect and gives a false impression that its cancer expression is much higher when summing all cancer counts from all tissues. Stratifying by tissue type guards against this bias.

\section{Heterogeneity of odds ratios}

In the strictest application, the use of Cochran-MantelHaenszel method requires the odds ratios of the sub-tables be homogeneous. In our context, it means the ratio of 
gene expressions between cancer and normal tissues are probably the same among all tissue types under study and any observed variability is most likely due to sampling bias. Also, the calculated odds ratio would be the estimated common odds ratio across the tissue strata. In our case, however, not all genes had similar ratios under each tissue (based on Woolf's test for homogeneity available in Additional file 2 under the "Woolf" column label), and this was of course expected. In spite of this, we were interested in the overall expression patterns of the genes in cancer conditions. We were not interested in an estimate of common odds ratio across the strata, which often does not exist. We were interested in hypothesis testing - to give us leads to the genes that had higher cancer expression in general. In this regard, the test could be applied [58,59]. The CMH odds ratio is a weighted average of the odds ratio in each tissue classification and can give us a summary measure [60], which we used to prioritize and followed up with subsequent biological analyses. In other word, an odds ratio in our data was merely a value that "average up" across all tissue types. From these ratios we were able to reveal the preferential cancer expressions, since the list covered a number of important known biomarkers, and enrichment of cancer-related genes were supported by knowledge-based GeneGO analyses and previous publications.

\section{Lower bound of confidence interval}

Another distinctive tactic we used is the selection of DEGs among the statistically significant genes ( $\mathrm{p}$-value < 0.05 ) base on lower bounds of the confidence interval of the odds ratio estimates. The popular approach to search for DEGs is to select genes base on p-value first, and then select the subset base on parameter estimators such as odds ratio or fold change values. The p-value criterion selects the statistically significant ones (those not likely to be the result of random fluctuation). The subsequent criterion is based on prior domain knowledge. However, among those with statistically significant $\mathrm{p}$-values and similar parameter estimators, the ranges of the estimations can vary widely. Using our dataset as an example, the two genes TUBA1B and FAM60A both have odd values of 2.38 (Additional file 2). However, for TUBA1B, it is within the $95 \%$ confidence that its true odds ratio is between 2.26 and 2.50. Yet for FAM60A it is between 1.59 and 3.54. Based on our background knowledge and for future application, if we must select genes having odds ratios greater than 2.0, then using odds ratio as cutoff would not serve this purpose since it is quite possible that the real odds ratio (i.e., of the population) is below 2.0. Choosing genes based on their confidence intervals would be more precise, but this has not been much appreciated.

\section{Multi-cancer biomarkers}

The multi-cancer approach compares genes that are overall differentially expressed among multiple cancer types comparing to their respective normal tissue types. Although many biomarker studies focus on gene differentially expressed in a particular tissue type, Wu et al. found 8 proteins in the conditioned media of 23 cell lines showing negative or weak tissue staining in the Human protein atlas, suggesting them to be potential pan-cancer markers [61]. Sahin et al., found that claudin-18 splice variant 2 had the ectopic activations in pancreatic, esophageal, ovarian, and lung tumors while its expression in normal tissue only occurred in differentiated epithelial cells of the gastric mucosa, confirmed by RT-PCR [62]. These studies suggested that relatively multi-cancer genes or multi-cancer splice variants exist. The three candidates COL3A1 (Collagen alpha-1(III) chain), DLG3 (Discs large homolog 3) (plasma membrane), and RNF43 (Ring finger protein 43) are putative secreted or plasma membrane proteins with the potential of developing serum diagnostic reagents. In reviewing the involvement of these genes with cancers in previous studies, hint for pan-cancer marker was surfaced as the expression of the extracellular matrix protein COL3A1 gene in brain cancer [63] and angiofibroma [64] was elevated. While secreted membrane bound RNF43 protein gene was known to be up-regulated in colorectal cancer [65]. Interestingly, upon the real-time qPCR analysis of three cancer differentially expressed secreted protein gene candidates, COL3A1, DLG3, and RNF43 identified in this study, higher cancer expression levels of these genes in multiple cancer types were verified. This does not only indicate the usefulness of our computational approach and filtering procedure but also encourages us to devote further resources for assessing the clinical usages of these three candidates.

\section{Pooling of gene expression}

Earlier in this discussion, we mentioned that naive pooling of data may introduce bias and at worst may produce Simpson's paradox. We also mentioned that we have tackled this problem with CMH. Nevertheless, two other occasions of pooling actually took place. We pooled expression from different splicing variants from the same gene to make one gene expression. We also pooled different libraries of the same tissue into one tissue classification. In both of these cases, we may encounter expression bias, since different splicing variants and different tissue libraries (i.e., tissues from different patients) might have differences in expression patterns. This is an unfortunate limitation in this and similar studies, since dbEST data consists of many different sources, and given the relative lack of data after the very stringent criteria we have used 
in our library selection compare to previous studies (Most importantly the exclusion of ESTs from cell lines, PCR amplification, subtraction, and cDNA normalization protocols). We opted for pooling since we had comparatively limited number of sequences to work with $(1,644,960$ out of $8,296,089$ downloaded - 18.03\%). Nonetheless, future digital expression profiling can be made better with the RNA-Seq methodology that offers a greater depth of coverage than ESTs obtained from traditional cDNA sequencing. It gives a much larger sampling size that makes more realistic the differentiation among isoforms and also makes pooling of different libraries of the same tissue less necessary. As for discovery of pan-cancer genes or isoforms when studying multiple tissue types, similar idea as outlined in this study would be just as applicable.

\section{Conclusions}

We have demonstrated that the use of the CochranMantel-Haenszel statistic in the integrative approaches allowed us to identify potential biomarkers or therapeutic targets via exhaustive search of various EST libraries from dbEST. As shown in previous study, splice variant could be useful target of antibody therapy [62]. The method can be easily extended over to searching cancer differential splicing variants had there been enough data. The issues involved in the analysis, such as the Simpson's paradox and the pan-cancer markers, may also be encountered in other multi-class digital analysis. The three targets confirmed by real-time qPCR, COL3A1, DLG3, and RNF43, are worthy of further evaluation for clinical applications.

\section{Additional material}

Additional file 1: Tissue and library distributions of 1,644,960 ESTs This table shows the number of ESTs assigned to each tissue type prior to matching to reference sequences.

Additional file 2: EST pipeline raw data. This is the raw EST count from the EST pipeline imported into Excel. The columns are the condition type, tissue, and condition-tissue type stratifications. The rows represent the EST counts that are assigned to genes.

\section{Acknowledgements}

This work was supported by grants NSC 99-3112-B-010-003 (W.V. Ng) from the National Science Council and an intramural grant derived from the Aim for the Top University Grant awarded to National Yang Ming University from the Ministry of Education, Taiwan, the Republic of China.

This article has been published as part of BMC Genomics Volume 13 Supplement 7, 2012: Eleventh International Conference on Bioinformatics (InCoB2012): Computational Biology. The full contents of the supplement are available online at http://www.biomedcentral.com/bmcgenomics/ supplements/13/S7.

\section{Author details}

'Institute of Biomedical Informatics, National Yang Ming University, Taipei, Taiwan, R.O.C. ${ }^{2}$ Molecular Medicine Research Center, Chang Gung University, Taoyuan, Taiwan, R.O.C. ${ }^{3}$ Department of Biotechnology and Laboratory
Science in Medicine and Institute of Biotechnology in Medicine, National Yang Ming University, Taipei, Taiwan, R.O.C. ${ }^{4}$ Bioinformatics Center, Chang Gung University, Taoyuan, Taiwan, R.O.C. ${ }^{5}$ Institute of Statistical Science, Academia Sinica, Taipei, 11529, Taiwan, R.O.C. ${ }^{6}$ Institute of Biomedical Sciences, Academia Sinica, Taipei, Taiwan, R.O.C. ${ }^{7}$ Center for Systems and Synthetic Biology, National Yang Ming University, Taipei, Taiwan, R.O.C.

\section{Authors' contributions}

TW and LC selected and filtered EST libraries as well as STRING analysis. TW conceived the deployment of the statistical method and implemented and ran the EST analysis pipeline. Microarray slides were selected by LC and analyzed by TW. TW, LC, TC were involved with literature search. LC selects the genes for expression validation and STRING analysis. JW performed the real-time $\mathrm{qPCR}$ analysis. $Y T$ is involved with the statistical interpretation of the results. WL and WN provided direction and guidance. All authors read and approved the final manuscript.

\section{Competing interests}

The authors declare that they have no competing interests.

Published: 13 December 2012

\section{References}

1. Pease AC, Solas D, Sullivan EJ, Cronin MT, Holmes CP, Fodor SP: Lightgenerated oligonucleotide arrays for rapid DNA sequence analysis. Proceedings of the National Academy of Sciences of the United States of America 1994, 91(11):5022-5026.

2. Schena M, Shalon D, Davis RW, Brown PO: Quantitative monitoring of gene expression patterns with a complementary DNA microarray. Science (New York, NY) 1995, 270(5235):467-470.

3. Casneuf T, Van de Peer Y, Huber W: In situ analysis of cross-hybridisation on microarrays and the inference of expression correlation. BMC Bioinformatics 2007, 8:461.

4. Okoniewski MJ, Miller CJ: Hybridization interactions between probesets in short oligo microarrays lead to spurious correlations. BMC Bioinformatics 2006, 7:276.

5. Adams MD, Kelley JM, Gocayne JD, Dubnick M, Polymeropoulos MH, Xiao H, Merril CR, Wu A, Olde B, Moreno RF, et al: Complementary DNA sequencing: expressed sequence tags and human genome project. Science (New York, NY) 1991, 252(5013):1651-1656.

6. Matsubara K, Okubo K: CDNA analyses in the human genome project. Gene 1993, 135(1-2):265-274.

7. Weinstock KG, Kirkness EF, Lee NH, Earle-Hughes JA, Venter JC: cDNA sequencing: a means of understanding cellular physiology. Curr Opin Biotechnol 1994, 5(6):599-603.

8. Adams MD, Kerlavage AR, Fleischmann RD, Fuldner RA, Bult CJ, Lee NH, Kirkness EF, Weinstock KG, Gocayne JD, White O, et al: Initial assessment of human gene diversity and expression patterns based upon 83 million nucleotides of cDNA sequence. Nature 1995, 377(6547 Suppl):3-174.

9. Ewing RM, Ben Kahla A, Poirot O, Lopez F, Audic S, Claverie JM: Large-scale statistical analyses of rice ESTs reveal correlated patterns of gene expression. Genome research 1999, 9(10):950-959.

10. Vasmatzis G, Essand M, Brinkmann U, Lee B, Pastan I: Discovery of three genes specifically expressed in human prostate by expressed sequence tag database analysis. Proceedings of the National Academy of Sciences of the United States of America 1998, 95(1):300-304.

11. Schmitt AO, Specht T, Beckmann G, Dahl E, Pilarsky CP, Hinzmann B, Rosenthal A: Exhaustive mining of EST libraries for genes differentially expressed in normal and tumour tissues. Nucleic acids research 1999, 27(21):4251-4260.

12. The Cancer Genome Anatomy Project cDNA xProfiler. [http://cgap.nci.nih. gov/Tissues/xProfiler].

13. NCBI Unigene Digital Differential Display. [http://www.ncbi.nlm.nih.gov/ UniGene/ddd.cgi].

14. The Cancer Genome Anatomy Project Digital Gene Expression Displayer.

15. Audic S, Claverie JM: The significance of digital gene expression profiles. Genome research 1997, 7(10):986-995.

16. Lal A, Lash AE, Altschul SF, Velculescu V, Zhang L, McLendon RE, Marra MA, Prange C, Morin PJ, Polyak K, et al: A public database for gene expression in human cancers. Cancer Res 1999, 59(21):5403-5407. 
17. Stekel DJ, Git Y, Falciani F: The comparison of gene expression from multiple cDNA libraries. Genome research 2000, 10(12):2055-2061.

18. Lu B, Xu J, Lai M, Zhang $H$, Chen J: A transcriptome anatomy of human colorectal cancers. BMC cancer 2006, 6:40.

19. Nie D, Xiang Y: Molecular cloning and characterization of a novel human testis-specific gene by use of digital differential display. Journal of genetics 2006, 85(1):57-62.

20. Clepet $C$, Joobeur $T$, Zheng $Y$, Jublot $D$, Huang $M$, Truniger $V$, Boualem $A$, Hernandez-Gonzalez ME, Dolcet-Sanjuan R, Portnoy V, et al: Analysis of expressed sequence tags generated from full-length enriched CDNA libraries of melon. BMC genomics 2011, 12:252.

21. Tang Q, Ma XJ, Mo CM, Wilson IW, Song C, Zhao H, Yang YF, Fu W, Qiu DY: An efficient approach to finding Siraitia grosvenorii triterpene biosynthetic genes by RNA-seq and digital gene expression analysis. BMC genomics 2011, 12(1):343.

22. Jager M, Ott CE, Grunhagen J, Hecht J, Schell H, Mundlos S, Duda GN Robinson PN, Lienau J: Composite transcriptome assembly of RNA-seq data in a sheep model for delayed bone healing. BMC genomics 2011 12:158.

23. Tillett RL, Ergul A, Albion RL, Schlauch KA, Cramer GR, Cushman JC: Identification of tissue-specific, abiotic stress-responsive gene expression patterns in wine grape (Vitis vinifera L.) based on curation and mining of large-scale EST data sets. BMC plant biology 2011, 11:86.

24. Kan Z, States D, Gish W: Selecting for functional alternative splices in ESTs. Genome research 2002, 12(12):1837-1845

25. Xu Q, Modrek B, Lee C: Genome-wide detection of tissue-specific alternative splicing in the human transcriptome. Nucleic acids research 2002, 30(17):3754-3766

26. Wang Z, Lo HS, Yang H, Gere S, Hu Y, Buetow KH, Lee MP: Computational analysis and experimental validation of tumor-associated alternative RNA splicing in human cancer. Cancer Res 2003, 63(3):655-657.

27. $\mathrm{Xu} \mathrm{Q}$, Lee $\mathrm{C}$ : Discovery of novel splice forms and functional analysis of cancer-specific alternative splicing in human expressed sequences. Nucleic acids research 2003, 31(19):5635-5643.

28. Hui L, Zhang X, Wu X, Lin Z, Wang Q, Li Y, Hu G: Identification of alternatively spliced mRNA variants related to cancers by genome-wide ESTs alignment. Oncogene 2004, 23(17):3013-3023.

29. Kirschbaum-Slager N, Parmigiani RB, Camargo AA, de Souza SJ: Identification of human exons overexpressed in tumors through the use of genome and expressed sequence data. Physiol Genomics 2005, 21(3):423-432.

30. He C, Zhou F, Zuo Z, Cheng H, Zhou R: A global view of cancer-specific transcript variants by subtractive transcriptome-wide analysis. PLoS One 2009, 4(3)::4732.

31. Valletti A, Anselmo A, Mangiulli M, Boria I, Mignone F, Merla G, D'Angelo V, Tullo A, Sbisa E, D'Erchia AM, et al: Identification of tumor-associated cassette exons in human cancer through EST-based computational prediction and experimental validation. Mol Cancer 2010, 9:230.

32. Rucker G, Schumacher M: Simpson's paradox visualized: the example of the rosiglitazone meta-analysis. BMC Med Res Methodol 2008, 8:34.

33. Mantel N, Haenszel W: Statistical aspects of the analysis of data from retrospective studies of disease. Journal of the National Cancer Institute 1959, 22(4):719-748.

34. Boguski MS, Lowe TM, Tolstoshev CM: dbEST-database for "expressed sequence tags". Nature genetics 1993, 4(4):332-333.

35. Dias Neto E, Correa RG, Verjovski-Almeida S, Briones MR, Nagai MA, da Silva W Jr, Zago MA, Bordin S, Costa FF, Goldman GH, et al: Shotgun sequencing of the human transcriptome with ORF expressed sequence tags. Proceedings of the National Academy of Sciences of the United States of America 2000, 97(7):3491-3496.

36. Jensen $L$, Kuhn M, Stark M, Chaffron S, Creevey C, Muller J, Doerks T, Julien P, Roth A, Simonovic M, et al: STRING 8-a global view on proteins and their functional interactions in 630 organisms. Nucleic acids research 2009, , 37 Database: D412-416.

37. Pruitt KD, Tatusova T, Klimke W, Maglott DR: NCBI Reference Sequences: current status, policy and new initiatives. Nucleic acids research 2009, 37 Database: D32-36.

38. NCBI RefSeq FTP. [ftp://ftp.ncbi.nih.gov/refseq/release/vertebrate_ mammalian].

39. RepeatMasker Open-3.0.1996-2010. [http://www.repeatmasker.org].

40. CGAP download site. [http://cgap.nci.nih.gov/Info/CGAPDownload]
41. Kent WJ: BLAT-the BLAST-like alignment tool. Genome research 2002, 12(4):656-664

42. Edgar R, Domrachev M, Lash AE: Gene Expression Omnibus: NCBI gene expression and hybridization array data repository. Nucleic acids research 2002, 30(1):207-210.

43. Bolstad B: Low Level Analysis of High-density Oligonucleotide Array Data: Background, Normalization and Summarization. University of California, Berkeley; 2004

44. Gautier L, Cope L, Bolstad BM, Irizarry RA: affy-analysis of Affymetrix GeneChip data at the probe level. Bioinformatics 2004, 20(3):307-315

45. Sean D, Meltzer PS: GEOquery: a bridge between the Gene Expression Omnibus (GEO) and BioConductor. Bioinformatics 2007, 23(14):1846-1847.

46. Smyth GK: Linear models and empirical bayes methods for assessing differential expression in microarray experiments. Stat Appl Genet Mol Biol 2004, 3:Article3.

47. Magrane M, Consortium U: UniProt Knowledgebase: a hub of integrated protein data. Database (Oxford) 2011, bar009.

48. Chen Y, Zhang Y, Yin Y, Gao G, Li S, Jiang Y, Gu X, Luo J: SPD-a webbased secreted protein database. Nucleic acids research 2005, 33 Database: D169-173.

49. Tusnady GE, Kalmar L, Simon I: TOPDB: topology data bank of transmembrane proteins. Nucleic acids research 2008, 36 Database: D234-239.

50. Sprenger J, Lynn Fink J, Karunaratne S, Hanson K, Hamilton NA, Teasdale RD: LOCATE: a mammalian protein subcellular localization database. Nucleic acids research 2008, , 36 Database: D230-233.

51. Tusnady GE, Dosztanyi Z, Simon I: Transmembrane proteins in the Protein Data Bank: identification and classification. Bioinformatics 2004, 20(17):2964-2972.

52. Tusnady GE, Dosztanyi Z, Simon I: PDB_TM: selection and membrane localization of transmembrane proteins in the protein data bank. Nucleic acids research 2005, 33 Database: D275-278.

53. Lomize MA, Lomize AL, Pogozheva ID, Mosberg HI: OPM: orientations of proteins in membranes database. Bioinformatics 2006, 22(5):623-625.

54. Raman P, Cherezov V, Caffrey M: The Membrane Protein Data Bank. Cell Mol Life Sci 2006, 63(1):36-51.

55. GeneGo. [http://www.genego.com/]

56. Smoot ME, Ono K, Ruscheinski J, Wang PL, Ideker T: Cytoscape 2.8: new features for data integration and network visualization. Bioinformatics 2011, 27(3):431-432.

57. Pearson K: On the criterion that a given system of deviations from the probable in the case of a correlated system of variables is such that it can be reasonably supposed to have arisen from random sampling. Philosophical Magazine, Series 5 1900, 50(302):157-175.

58. Greenland S: Interpretation and estimation of summary ratios under heterogeneity. Statistics in medicine 1982, 1(3):217-227.

59. McDonald JH: Handbook of Biological Statistics. Sparky House Publishing Baltimore, Maryland; 2 2nd 2009 .

60. Kuritz SJ, Landis JR, Koch GG: A general overview of Mantel-Haenszel methods: applications and recent developments. Annual review of public health 1988, 9:123-160

61. Wu CC, Hsu CW, Chen CD, Yu CJ, Chang KP, Tai DI, Liu HP, Su WH, Chang YS, Yu JS: Candidate serological biomarkers for cancer identified from the secretomes of 23 cancer cell lines and the human protein atlas. Molecular \& cellular proteomics : MCP 2010, 9(6):1100-1117.

62. Sahin U, Koslowski M, Dhaene K, Usener D, Brandenburg G, Seitz G, Huber C, Tureci O: Claudin-18 splice variant 2 is a pan-cancer target suitable for therapeutic antibody development. Clinical cancer research an official journal of the American Association for Cancer Research 2008, 14(23):7624-7634.

63. Liu Y, Carson-Walter EB, Cooper A, Winans BN, Johnson MD, Walter KA: Vascular gene expression patterns are conserved in primary and metastatic brain tumors. Journal of neuro-oncology 2010, 99(1):13-24.

64. Gramann M, Wendler O, Haeberle L, Schick B: Expression of collagen types I, II and III in juvenile angiofibromas. Cells, tissues, organs 2009, 189(6):403-409.

65. Yagyu R, Furukawa $Y$, Lin YM, Shimokawa T, Yamamura T, Nakamura Y: A novel oncoprotein RNF43 functions in an autocrine manner in colorectal cancer. International journal of oncology 2004, 25(5):1343-1348.

66. Mok SC, Bonome T, Vathipadiekal V, Bell A, Johnson ME, Wong KK, Park DC, Hao K, Yip DK, Donninger $\mathrm{H}$, et al: A gene signature predictive for 
outcome in advanced ovarian cancer identifies a survival factor: microfibril-associated glycoprotein 2. Cancer cell 2009, 16(6):521-532.

67. Bowen NJ, Walker LD, Matyunina LV, Logani S, Totten KA, Benigno BB, McDonald JF: Gene expression profiling supports the hypothesis that human ovarian surface epithelia are multipotent and capable of serving as ovarian cancer initiating cells. BMC medical genomics 2009, 2:71.

68. Badea L, Herlea V, Dima SO, Dumitrascu T, Popescu I: Combined gene expression analysis of whole-tissue and microdissected pancreatic ductal adenocarcinoma identifies genes specifically overexpressed in tumor epithelia. Hepato-gastroenterology 2008, 55(88):2016-2027.

doi:10.1186/1471-2164-13-S7-S12

Cite this article as: Wu et al:: Meta-analytical biomarker search of EST expression data reveals three differentially expressed candidates. BMC Genomics 2012 13(Suppl 7):S12.

\section{Submit your next manuscript to BioMed Central} and take full advantage of:

- Convenient online submission

- Thorough peer review

- No space constraints or color figure charges

- Immediate publication on acceptance

- Inclusion in PubMed, CAS, Scopus and Google Scholar

- Research which is freely available for redistribution

Submit your manuscript at www.biomedcentral.com/submit
C Biomed Central 